\title{
Roche
}

\section{Real-time, label-free monitoring of cellular invasion and migration with the xCELLigence system}

\begin{abstract}
For researchers performing invasion and migration assays, the CIM-Plate 16 used with the xCELLigence RTCA DP Instrument from Roche Applied Science offers a means to monitor cellular responses in real time, without exogenous labels, through impedance-based technology. The CIM-Plate 16 provides a kinetic cell-response profile throughout an experiment, detailing the onset and rate of invasion and migration. This information can help researchers understand processes that current endpoint experiments are unable to address.
\end{abstract}

Principles of invasion and migration for cellular analysis The study of cell invasion and migration is of great importance to enable a better understanding of underlying biological and molecular mechanisms. The new CIM-Plate 16 device from Roche Applied Science detects real-time invasion and migration of cells without the use of labels.

Cell invasion is the intrusion on and destruction of adjacent tissues, particularly with respect to cancer cells. Cell migration is the movement of cells from one area to another, generally in response to chemical signal, and is important in diverse physiological and pathological processes including embryonic development, cell differentiation, wound healing, immune response, inflammation and, notably, cancer metastasis.

Conventional methodologies for migration detection are endpoint and label-based assays. One of the more commonly used methods is the Boyden chamber (also called trans-well migration assay), whereby cells placed in inserts are allowed to invade and migrate through an artificial microporous membrane into lower chambers containing a given chemoattractant.

There are drawbacks to this approach. The migrated cells (typically attached on the reverse side of the membrane) must be stained with chemical dyes or labeled with fluorescent molecules. In most cases, the membrane is removed from the insert, and then the stained or labeled cells are counted manually using a microscope or evaluated using a spectrometer. This labor-intensive process limits throughput and, as a result of labeling the cells, may alter gene expression profiles and lead to intra-assay variability because of differences in labeling efficiency. Additionally, cell counting by means of microscopy may not be accurate, often resulting in inconsistent and irreproducible migration data.

\section{Christopher Bird \& Shelli Kirstein}

Roche Diagnostics, Roche Applied Science, Indianapolis, Indiana, USA. Correspondence should be addressed to S.K. (shelli.kirstein@roche.com).

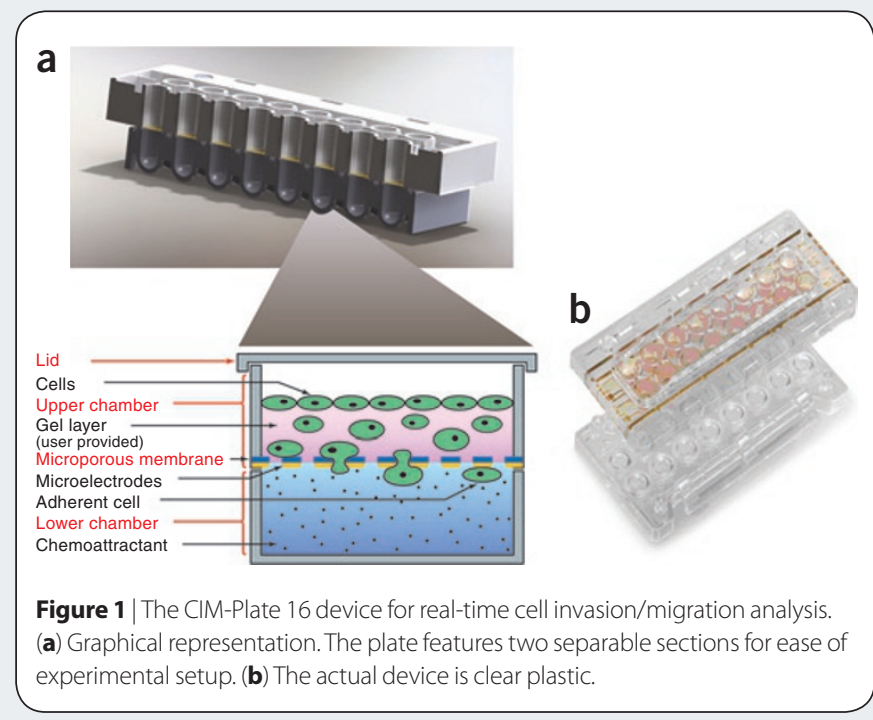

CIM-Plate 16 detects cell invasion/migration in real time In contrast to the methods mentioned above, the Roche xCELLigence Real-Time Cell Analyzer (RTCA) DP instrument provides kinetic information about cell migration by dynamically recording the entire cell migration and invasion process in real time without labeling cells, considerably improving invasion and migration assay quality. The RTCA DP instrument uses the CIM (cellular invasion/ migration)-Plate 16, which features microelectronic sensors integrated onto the underside of the microporous polyethylene terephthalate (PET) membrane of a Boyden-like chamber (Fig. 1). As cells migrate from the upper chamber through the membrane into the bottom chamber in response to chemoattractant, they contact and adhere to the electronic sensors on the underside of the membrane, resulting in an increase in impedance ${ }^{1}$. The impedance increase correlates to increasing numbers of migrated cells on the underside of the membrane, and cell-index values reflecting impedance changes are 


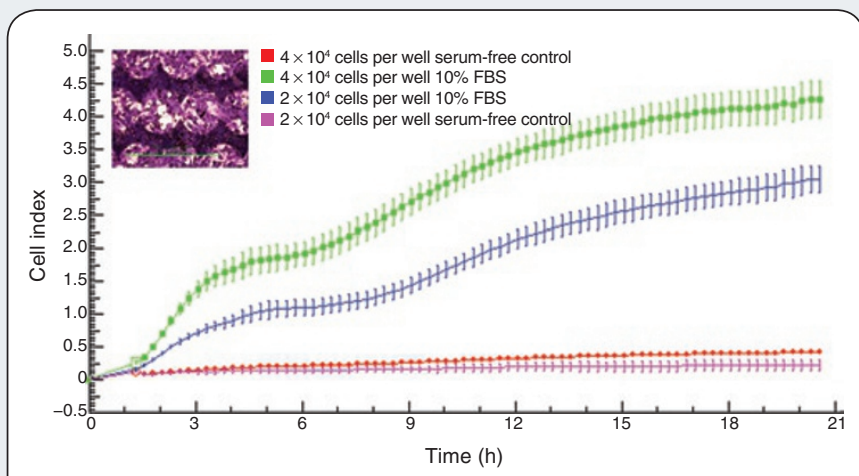

Figure 2 | Migration kinetics of HeLa cells, as shown by continuous monitoring of live-cell migration. Results shown are mean \pm s.d. $(n=4)$. Inset, a representative image of the migrated cells stained (using a Diff-Quick staining kit; Fisher) on the underside of the membrane upon completion of the experiment. Scale bar, 1,630 $\mu \mathrm{m}$.

automatically and continuously recorded by the RTCA DP instrument (Figs. $\mathbf{2}$ and $\mathbf{3}$ ). Therefore, cell migration activity can be monitored via the cell-index profile.

\section{Experimental design}

The following is a basic migration assay protocol used on the RTCA DP instrument, with fetal bovine serum (FBS) as chemoattractant. The protocol is optimized for two cancer cell lines: a human fibrosarcoma cell line, HT1080, and a human cervical cancer cell line, HeLa. Assay conditions may require further optimization if different types of cell lines or chemoattractants are used. (i) Cells should be grown in serumfree medium for 4-24 hours. (ii) For invasion experiments, the top chamber of the CIM-Plate 16 is coated with Matrigel (BD) before adding medium (with or without chemoattractant) to the bottom chamber. (iii) The CIM-Plate 16 is assembled by placing the top chamber onto the bottom chamber and snapping the two together. (iv) Serum-free medium is placed in the top chamber to hydrate and preincubate the membrane for 1 hour in the $\mathrm{CO}_{2}$ incubator at $37{ }^{\circ} \mathrm{C}$ before obtaining a background measurement. (v) Cells are lightly trypsinized, pelleted and resuspended at the indicated cell densities in serum-free medium. (vi) Once the CIM-Plate 16 has equilibrated, it is placed in the RTCA DP station and the background cell-index values are measured. (vii) The CIM-Plate 16 is then removed from the RTCA DP station and the cells are added to the top chamber at the desired density. (viii) The CIM-Plate 16 is placed in the RTCA DP station and migration is monitored every 2 minutes for several hours.

\section{Migration of HeLa cells}

We analyzed two densities of HeLa cells in the absence or presence of $10 \%$ FBS in the bottom chamber (Fig. 2). Cell migration kinetics were recorded by the RTCA DP instrument for approximately 18 hours. Migration kinetics were distinct and easily detected by automated realtime monitoring. The low and high seeding densities were quantitatively monitored and reflected by the cell-index values, and were correlated with a manual cell count determined at the end of the assay by fixing and staining the cells on the membrane of the top chamber.

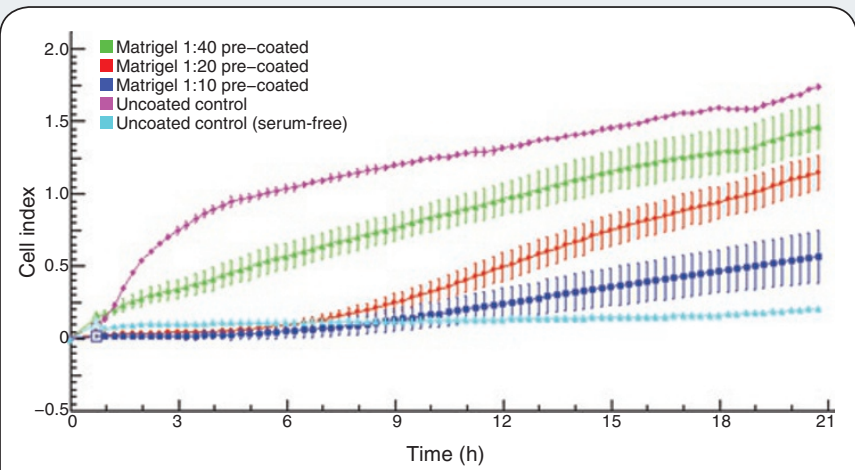

Figure 3 | Invasion and migration kinetics of HT1080 cells in indicated dilutions of Matrigel using FBS as the chemoattractant. HT1080 cells were seeded at $2 \times 10^{4}$ cells per well in either uncoated wells (migration) or Matrigel-coated wells (invasion). Results shown are mean \pm s.d. $(n=4)$. Precoating was done at $37^{\circ} \mathrm{C}$ for 4 hours.

\section{Invasion and migration of HT1080 cells}

We monitored migration and invasion kinetics - in the absence and presence of Matrigel, respectively_in response to 10\% FBS (Fig. 3). In the absence of serum, we observed little to no migration. As indicated by the cell-index profiles, cell invasion and migration was affected in a dose-dependent manner by the concentration of Matrigel. The rate and onset of invasion were readily quantified by real-time monitoring while concurrently assessing migration. The delay of cell-index signal (Fig. 3) was due to the time required for the cells to invade through the Matrigel and was dependent on the concentration of Matrigel used.

\section{Conclusion}

The cancerous cells shown have varying migratory and invasive behaviors, specific to chemoattractant stimuli or matrigel concentration. The new CIM-Plate 16 for the RTCA DP instrument is a versatile device for the analysis of invasion and migration, and is at present the only platform capable of quantitatively measuring the invasive and migratory behavior of cells in real time without exogenous labels. This enables the identification of migratory and invasive time points, which will help increase throughput by shortening assay time, minimize the number of endpoint assays and help determine optimal time points for inhibitor studies. Moreover, analysis using the xCELLigence System reduces hands-on cellmanipulation steps, leading to more physiologically relevant results.

The CIM-Plate 16 will be available in the fall of 2009. More information about the xCELLigence System is available at http://www. xcelligence.roche.com/.

For life science research only. XCELLIGENCE is a trademark of Roche. Matrigel is a trademark of Becton, Dickinson and Company. Other brands or product names are trademarks of their respective holders.

1. Solly, K., Wang, X., Xu, X., Strulovici, B. \& Zheng,W. Application of real-time cell electronic sensing (RT-CES) technology to cell-based assays. Assay Drug Dev. Technol. 2, 363-372 (2004)

This article was submitted to Nature Methods by a commercial organization and has not been peer reviewed. Nature Methods takes no responsibility for the accuracy or otherwise of the information provided. 